\title{
High stakes anesthesia: Anesthetic considerations and implications for complete dental extraction in a patient with complex comorbidities
}

\author{
Rohan K. Panchamia, Jon D. Samuels \\ Department of Anesthesiology, Weill Cornell Medicine, New York, NY, United States
}

\begin{abstract}
This case report describes a frail, middle-aged woman with multiple comorbidities who was scheduled to undergo extraction of all remaining teeth in anticipation of cardiac quadruple valve intervention. Dental and anesthetic management of the patient are discussed. Medical care of the patient with a high burden of comorbidities requires a multidisciplinary approach even for a routine dental procedure.
\end{abstract}

Keywords: Comorbidity; General Anesthesia; Heart Valve Diseases; Tooth Extraction.

This is an Open Access article distributed under the terms of the Creative Commons Attribution Non-Commercial License (http://creativecommons.org/licenses/by-nc/4.0/) which permits unrestricted non-commercial use, distribution, and reproduction in any medium, provided the original work is properly cited.

\section{INTRODUCTION}

The patient with complex comorbidities having dental surgery would benefit from a multidisciplinary approach. This is true even for minor dental procedures, e.g. dental extraction. Decision of the type of anesthesia for minor dental procedures, whether monitored anesthesia care (MAC) or general anesthesia, is made jointly by the dentist or oral surgeon, the anesthesiologist, and the patient. Given the risks and complications associated with the use of general anesthetics, almost all potentially painful oral and maxillofacial procedures have been performed under MAC whenever feasible [1]. General anesthesia has been strictly limited to certain patients and clinical situations in which local anesthesia is not an option.

\section{CASE DETAILS}

A 57-year-old woman (height $164 \mathrm{~cm}$, weight $62.5 \mathrm{~kg}$, BMI $23 \mathrm{~kg} / \mathrm{m}^{2}$ ) with multiple comorbidities, the most serious of which were her multivalvular insufficiency with biventricular dysfunction and severe pulmonary hypertension, as well as severe persistent asthma, was electively scheduled to undergo extraction of 20 remaining teeth in anticipation of quadruple valve intervention. Cardiac surgery would not occur until after dental rehabilitation. A preoperative dental exam revealed generalized gross caries of remaining dentition, multiple fractured teeth, and generalized chronic periodontitis.

Her extensive past medical history included congenital heart disease (atrial septal defect and pulmonary stenosis) status post-surgical repair at the age of 12 . More recently, she developed multiple cardiac valvulopathies with

Received: April 4, 2019 - Revised: May 7, 2019 - Accepted: May 17, 2019

Corresponding Author: Rohan K. Panchamia, Department of Anesthesiology ,Weill Cornell Medical College, 525 E 68 th St. Box 124 New York New York 10065 , United States

Tel: +212-746-2972 E-mail: rkp9004@med.cornell.edu, rohanp26@gmail.com

Copyright(c) 2019 Journal of Dental Anesthesia and Pain Medicine 


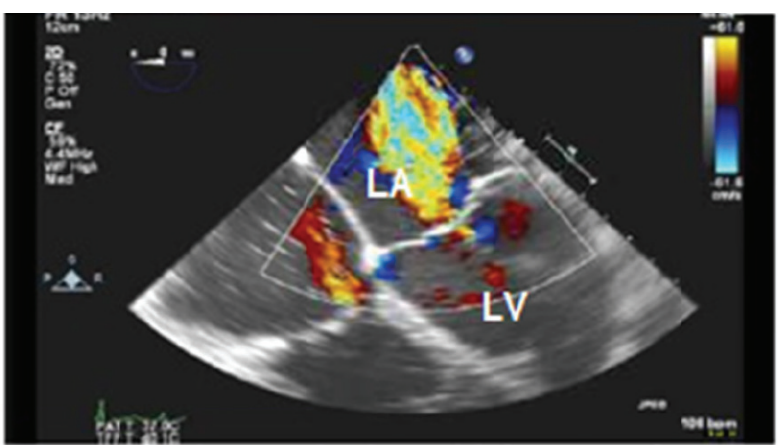

Severe mitral regurgitation with jet area of $10 \mathrm{~cm}^{2}$

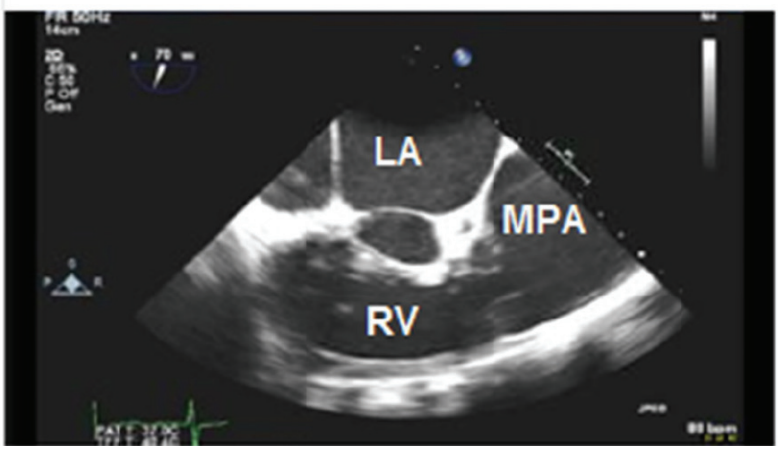

Severely dilated pulmonary artery

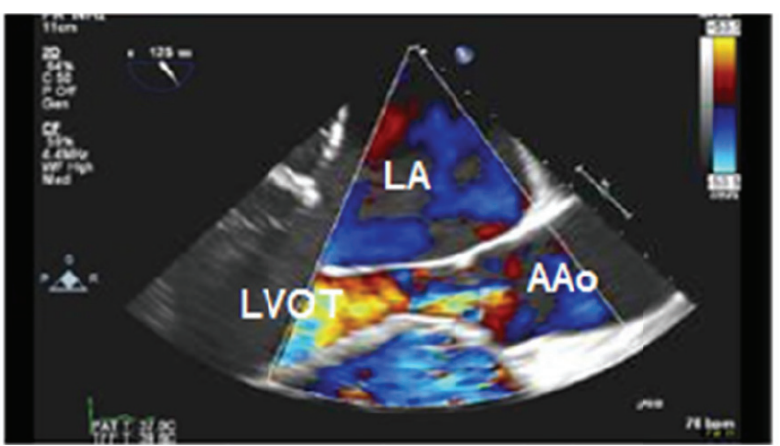

Moderate aortic regurgitation

Fig. 1. Preoperative TEE depicting severe MR, moderate AR, and a severely dilated pulmonary artery.

resulting biventricular failure and World Health Organization (WHO) group II severe pulmonary hypertension (pHTN). Her functional status was poor characterized by a metabolic equivalent of functional capacity (METS) of less than 3 METS; frequent episodes of shortness of breath occasionally accompanied by wheezing, and anxiety secondary to "air hunger."

Preoperative transesophageal echocardiogram (TEE) revealed severe mitral regurgitation (MR), mixed stenotic and regurgitant pathology of remaining cardiac valves, and depressed biventricular function (Fig. 1). Right heart catheterization was notable for elevated filling pressures and a PA pressure of 85/30/52 (mean) mmHg. Of note, during her heart catheterization less than 1 year prior, she went into flash pulmonary edema from anxietyinduced hypertensive crisis, requiring admission to the ICU and management with non-invasive positive pressure ventilation, nitroglycerine, and milrinone infusions. At the time of evaluation, she had compensated congestive heart failure on her neurohormonal antagonists, i.e. furosemide, lisinopril, and spironolactone.

Her asthma was classified as severe persistent, interfering with daily activities secondary to increased work of breathing, with multiple exacerbations requiring intubation, despite being on fluticasone propionate/ salmeterol and tiotropium bromide inhalers, taking oral prednisone, and doing standing albuterol nebulizer treatments 4 times daily. CT chest without contrast showed marked enlargement of the main PA and mild dilatation of the ascending aorta (Fig. 2), as well as diffuse ground-glass opacities and pulmonary nodules (Fig. 3), consistent with interstitial lung disease, possibly from prior asbestos exposure. CXR demonstrated cardiomegaly with enlarged pulmonary arteries; no effusion. Most recent PFTs were significant for a severe restrictive pattern, likely influenced by her cardiomegaly.

She was bridged from warfarin to enoxaparin for thromboprophylaxis (CHA2DS2-VASc $=6) 3$ days prior to surgery, given her longstanding history of atrial fibrillation, complicated by an ischemic cerebrovascular 


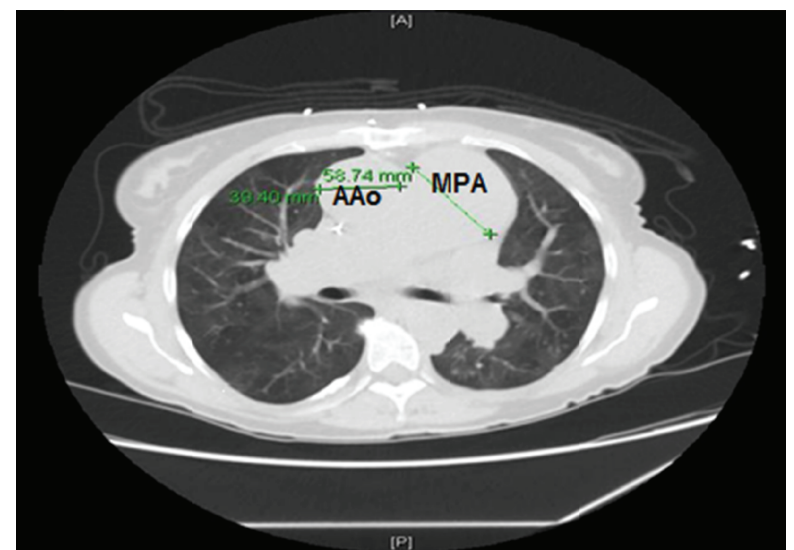

Fig. 2. Severe dilation of main pulmonary artery and mild dilation of ascending aorta as seen on CT chest without contrast.

accident without any residual deficits. A preoperative ECG showed atrial fibrillation with premature ventricular complexes; diffusely unchanged ST-T abnormalities compared to prior studies. On the day of surgery, physical examination was significant for a difficult airway: modified Mallampati class (MMC) 4 airway along with decreased thyromental $(25 \mathrm{~mm})$ and inter-incisor $(22 \mathrm{~mm})$ distances.

The patient entered a pre-warmed OR given a history of cold agglutinin disease. We administered midazolam $4 \mathrm{mg}$ total (in divided doses) intravenously for anxiolysis and amnesia. She received a radial arterial line for beat-to-beat blood pressure monitoring and arterial blood gas determination. We instructed her to take two puffs of albuterol from a metered dose inhaler (MDI) in order to relax airway smooth muscle prior to airway instrumentation. We then gave a stress dose of hydrocortisone $100 \mathrm{mg}$ given her steroid dependency. Following this, we de-nitrogenated her with $100 \%$ oxygen by mask in a semi-recumbent position. Fentanyl $50 \mathrm{mcg}$ was administered to suppress airway reflexes and prevent sympathetic activation caused by intubation. She received intravenous lidocaine $60 \mathrm{mg}(\sim 1 \mathrm{mg} / \mathrm{kg})$ to promote bronchodilation and prevent reflex bronchospasm. We induced general anesthesia with etomidate $12 \mathrm{mg}(\sim 0.2$ $\mathrm{mg} / \mathrm{kg}$ ) to minimize cardiac depression. After mask ventilation was established, we gave rocuronium $35 \mathrm{mg}$ $(\sim 0.6 \mathrm{mg} / \mathrm{kg})$ for skeletal muscle relaxation. To achieve

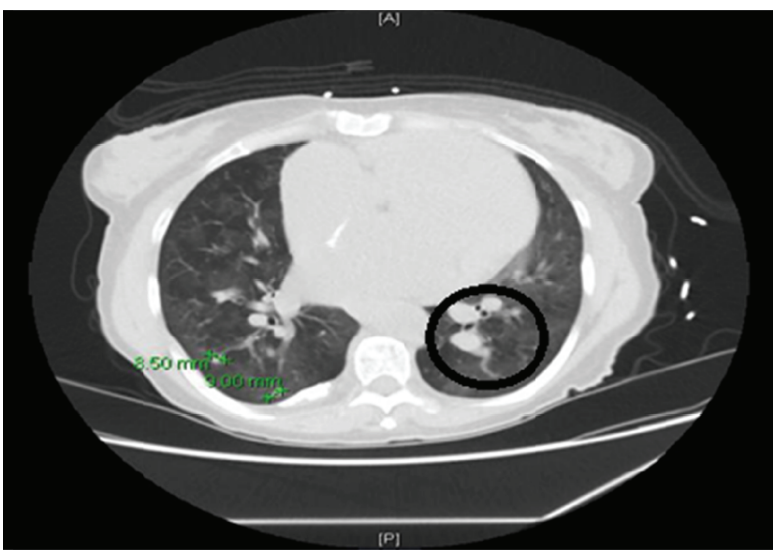

Fig. 3. Multiple ground-glass opacities and pulmonary nodules (circular region) on $\mathrm{CT}$ chest without contrast.

a deep plane of anesthesia and sustained bronchodilation, we delivered a high-inspired concentration $(2.5 \%)$ of sevoflurane anesthesia to the patient during mask ventilation. We successfully performed gentle laryngoscopy with the AirtraqTM SP (Prodol Meditec, Vizcaya, Spain) and inserted an oral 6.5 ETT. A flexible fiberoptic bronchoscope (Karl Storz Endoscopy, Tuttlingen, Germany) was available for back-up airway management.

Due to the patient being on anticoagulation with an INR of 1.8 , we did not attempt a nasal intubation. We did not place a TEE probe, as the operative site was the oral cavity; moreover, a previous TEE exam within the past year resulted in an esophageal tear, making blind insertion ill- advised. Transthoracic echocardiogram (TTE) was available in the event of hemodynamic instability but not needed. We had a pulmonary artery catheter in the room and decided we would only place it for determination and management of a shock state. Furthermore, her right-sided insufficiency, i.e. TR, provided no guarantee that we would be successful in placing one. The patient had no indication for infective endocarditis prophylaxis since the congenital defects were repaired.

We maintained heart rate and arterial blood pressure measurement at the patient's preoperative baseline with a low-dose infusion of phenylephrine (started shortly following intubation) and periodic administration of glycopyrrolate. Periodic glycopyrrolate boluses acted as 
a drying agent, improving operative conditions, minimized upper airway autonomic vagal reflexes, and reversed the mild reflex bradycardia of phenylephrine. Alternatively, dobutamine could have been used. Tight hemodynamic goals were set at a HR of $80-100 / \mathrm{min}$, favorable to regurgitant valvular lesions, with mean arterial pressures (MAPs) 65-80 mmHg for right heart perfusion.

Due to this patient's severe intrinsic asthma, we elected to base the maintenance anesthetic on an inhalation technique, using the volatile agent with most favorable smooth muscle relaxant properties, sevoflurane. Anesthesia for dental extraction generally does not require large doses of narcotics in the presence of alveolar nerve blocks, so remifentanil was not chosen; it would have likely cut down on the sevoflurane dosage, increasing the chance of intraoperative bronchospasm, as well as increasing the cost of the anesthetic.

Ventilation parameters allowed for adequate time in expiration and decreased risk of atelectasis. Arterial blood gases were unremarkable for acidosis, hypercarbia, hypoxia, or electrolyte abnormalities. Duration of surgery was approximately 150 minutes. The operative course, including extubation, was uneventful. The patient was discharged home from the ICU on postoperative day \#2.

\section{DISCUSSION}

Safe provision of dental services required the independent presence of an anesthesiologist due to the tenuous condition of this patient. We selected general anesthesia for two reasons: 1. the patient's history of crippling anxiety, which likely precipitated flash pulmonary edema during her cardiac catheterization, and 2. her multiple comorbidities, any one of which could have progressed to life-threatening decompensation or crisis unless meticulously managed.

A significant health problem among the elderly, the prevalence of congestive heart failure (CHF) is increasing with the aging demographic of the United States [2]. Due to diminished cardiac function, congestive heart failure represents a significant risk for perioperative morbidity and mortality in patients undergoing surgery [3]. Given possible reduced cardiac output in patients with $\mathrm{CHF}$, optimization of hemodynamics is critical in the anesthetic management of these patients. CHF patients are commonly treated with a variety of medications, such as beta blockers, diuretics, and angiotensin converting enzyme inhibitors (ACE-I) [4].

Substantial controversy exists regarding recommendations on the administration of ACE-I on the day of surgery. This issue is far from resolved. Most of the publications in this regard are retrospective observational studies, prone to potential confounders. On the hold side, patients receiving ACE-I on the day of surgery have a higher incidence of hypotension, which may not respond to commonly used sympathomimetics, such as phenylephrine or ephedrine. On the administer side, hypotension readily responds to vasopressin, which was ready, but not needed in our patient. The official position of the American Society of Anesthesiologists (ASA) is that this question should be handled on a case-by-case basis, and that the risk, while present, is small and that when given surgery should not be canceled. We discussed this issue with the dentist and patient, and decided to continue the ACE-I in the setting of stable preoperative hemodynamics in a patient scheduled for a minor surgical procedure [5]. Pulmonary hypertension results from a pathological increase in pulmonary vascular resistance (PVR) that leads to decreased flow through the pulmonary circulation and possibly RV dysfunction. The patient's pHTN fell into the WHO group II category, meaning it was caused by an increase in left-sided pressures (due to left heart pathology) [6]. She had several independent predictors of morbidity and mortality in patients with pHTN undergoing non-cardiac surgery, including higher ASA class, longer duration of surgery and anesthesia $(>3$ hours), chronic renal insufficiency, and New York Heart Association functional class $\geq 2$ [7]. Our perioperative ventilation strategy incorporated a high inspired fraction of oxygen, respiratory rate titrated to mild hypocapnia, optimum level of PEEP $\left(5-8 \mathrm{~cm} \mathrm{H}_{2} \mathrm{O}\right)$, and reasonable 
tidal volume (6-8 $\mathrm{cc} / \mathrm{kg}$ of predicted body weight), as to not cause atelectasis or hyperinflation, both of which can exacerbate PVR. Management of hemodynamics focused on maintaining sinus rhythm; adequate MAPs for RV coronary perfusion pressure; and judicious fluid administration to avoid RV underfilling or overdistension. Stressors of pHTN, such as hypoxemia, hypercapnia, hypothermia, acidosis, pain, and anxiety were actively prevented to avoid acute rises in PA pressures.In the setting of a surgery, IV anesthetic agents can increase the risk of hypotension through their effects on the sympathetic nervous system. Care must also be exercised during the induction of anesthesia as propofol could result in profound cardiac depression [8]. Furthermore, propofol increases arterial compliance, leading to further reduction in system blood pressure. We decided to induce with etomidate since there is less myocardial and autonomic depression associated with its administration. Intraoperatively, hypotension may be addressed with fluids, a sympathomimetic or an alpha-agonist, such as phenylephrine-our pressor of choice. Inotropic agents, such as dobutamine and epinephrine, may be used along with vasodilators, such as sodium nitroprusside, to increase the cardiac output of CHF patients undergoing surgery. Monitoring also plays a critical role in the management of patients with CHF. Arterial lines and pulmonary artery catheters can assess a patient's response to fluids, anesthetic agents and sympathomimetics [9].

Risk of pulmonary complications is greater in asthmatics with recent exacerbations, prior postoperative pulmonary complications, recent hospitalizations, or intubations for asthma. Surprisingly, an arterial blood gas, chest radiograph or pulmonary function test results are not predictive of complications [10]. Bronchoconstriction can occur in asthmatic patients undergoing anesthesia, which may be present as hypoxemia or difficulty in ventilation [11]. The mechanical stimulation that occurs during intubation may lead to bronchospasm and must be avoided [11]. Suppression of airway reflexes can be achieved with agents that dilate the bronchioles, such as propofol [12]. Intravenous lidocaine may be added to relax the smooth muscle of the airways and mitigate the response to intubation [13]. A volatile anesthetic based technique is recommended in this case, and selection of the particular agent is important in reducing the likelihood of bronchospasm. Sevoflurane, the volatile anesthetic which we selected, in contrast to others, e.g. desflurane, has proven to be less irritating to the airways and less likely to cause bronchoconstriction [14]. In contrast, a total intravenous anesthetic (TIVA), is not recommended, as bronchial tone is unabated. Laryngeal mask airways have also been shown to cause less bronchoconstriction compared to endotracheal intubation, but cannot be utilized here, as they block the surgical field [14]. To maximize ventilation and reduce air trapping, both the inspiratory and expiratory flow rates should be slowed. To reduce the viscosity of airway secretions, the patient should be well-hydrated both pre- and intra-operatively. A volatile anesthetic based technique is recommended in this case.

Valvular heart disease can lead to either pressure overload, such as in cases of AS, or volume overload, such as in cases of MR [15]. Pressure overload leads to concentric ventricular hypertrophy, which causes impairments in ventricular relaxation and elevated end-diastolic pressures [9]. Due to the diastolic dysfunction associated with pressure overload, patients require sufficient volume to fill the hypertrophied ventricle and thus blood pressure must be maintained [16]. Reductions in blood pressure can reduce coronary perfusion and precipitate myocardial ischemia [17]. Tachycardia should be avoided to promote ventricular filling during diastole and coronary perfusion [18]. In contrast to cases of pressure overload, volume overload occurs in cases of valvular insufficiency such as MR [17]. This is characterized by a decreased cardiac output secondary to a compromised forward flow. To promote forward flow in these patients, preload and contractility should be maintained while afterload should be reduced, such as with nitroprusside, hydralazine or ACE-I [15,18-19]. Bradycardia should be avoided as it can lead to exacerbations in volume overload. [16].

Although most dental extractions may be managed with 
local anesthesia administered by our dental colleagues, a few select cases require the services of an anesthesiologist: extensive or complex dental procedures, special needs patients, pediatric patients, severe TMJ dysfunction, facial abscesses, and the rare allergy to local anesthetics. The vast majority of dental procedures requiring an anesthesiologist will necessitate general endotracheal anesthesia with a nasal approach preferred over an oral route. Anticoagulation is a contraindication to nasal intubation. When the patient with a difficult airway presents for dental surgery, these are challenging cases. Our patient highlights the difficulties encountered with a patient with a heavy burden of comorbidities, therapeutic anticoagulation and a difficult airway.

\section{AUTHOR ORGIIS}

Rohan K. Panchamia: https://orcid.org/0000-0003-4892-6788

Jon D. Samuels: https://orcid.org/0000-0002-7040-1366

CONFLICT OF INTEREST: None of the authors have any conflicts of interest to declare.

\section{REFERENCES}

1. Cantlay K, Williamson S, Hawkings J. Anaesthesia for dentistry. Cont Edu Anaesth Crit Care Pain 2005; 5: 71-75.

2. Croft JB, Giles WH, Pollard RA, Keenan NL, Casper ML, Anda RF. Heart failure survival among older adults in the United States: a poor prognosis for an emerging epidemic in the Medicare population. Arch Intern Med 1999; 159: 505-10.

3. Goldman L. Cardiac risk in noncardiac surgery: an update. Anesth Analg 1995; 80: 810-20.

4. Ramani GV, Uber PA, Mehra MR. Chronic heart failure: contemporary diagnosis and management. Mayo Clin Proc 2010; 85: 180-95.

5. Roshanov PS, Rochwerg B, Patel A, Salehian O, Duceppe E, Belley-Côté EP, et al. Withholding versus continuing angiotensin-converting enzyme inhibitors or angiotensin
II receptor blockers before noncardiac surgery: an analysis of the vascular events in noncardiac surgery patients cohort evaluation prospective cohort. Anesthesiology 2017; 126: 16-27.

6. Simonneau G, Robbins IM, Beghetti M, Channick RN, Delcroix M, Denton CP, et al. Updated clinical classification of pulmonary hypertension. J Am Coll Cardiol 2009; 54(1 Suppl): S43-54.

7. D’Alonza GE, Barst RJ, Ayres SM, Bergofsky EH, Brundage BH, Detre KM, et al. Survival in patients with primary pulmonary hypertension. Results from a national prospective registry. Ann Intern Med 1991; 115: 343-9.

8. Win NN, Fukayama, H, Kohase, H, Umino, M. The different effects of intravenous propofol and midazolam sedation on hemodynamic and heart rate variability. Anesth Analg 2005; 101: 97-102.

9. Mebazaa A, Pitsis AA, Rudiger A, Toller W, Longrois D, Ricksten SE, et al. Clinical review: practical recommendations on the management of perioperative heart failure in cardiac surgery. Crit Care 2010; 14: 201.

10. Johnson RG, Arozullah AM, Neumayer L, Henderson WG, Hosokawa P, Khuri SF. Multivariable predictors of postoperative respiratory failure after general and vascular surgery: results from the patient safety in surgery study. J Am Coll Surg 2007; 204: 1188-98.

11. Tirumalasetty J, Grammer LC. Asthma, surgery, and general anesthesia: a review. J Asthma 2006; 43: 251-4.

12. Pizov R, Brown RH, Weiss YS, Baranov D, Hennes H, Baker S, et al. Wheezing during induction of general anesthesia in patients with and without asthma. A randomized, blinded trial. Anesthesiology 1995; 82: 1111-16.

13. Adamzik M, Groeben H, Farahani R, Lehmann N, Peters J. Intravenous lidocaine after tracheal intubation mitigates bronchoconstriction in patients with asthma. Anesth Analg 2007; 104: 168-72.

14. Woods BD, Sladen RN. Perioperative considerations for the patient with asthma and bronchospasm. Br J Anaesth 2009; 103(1 Suppl): i57-65.

15. Herrara A. Valvular heart disease. In: Stoelting's Anesthesia and Co-Existing Disease. 6th ed. Edited by Hines RL, Marschall KE. Philadelphia, Elsevier(Saunders). 2012, pp 
$31-47$.

16. Frogel J, Galusca D. Anesthetic considerations for patients with advanced valvular heart disease undergoing noncardiac surgery. Anesthesiol Clin 2010; 28: 67-85.

17. Enriquez-Sarano M, Akins CW, Vahanian A. Mitral regurgitation. Lancet 2009; 373: 1382-94.
18. Stone JG, Faltas AN, Hoar PF. Sodium nitroprusside therapy for cardiac failure in anesthetized patients with valvular insufficiency. Anesthesiology 1978; 49: 414-18.

19. Boon NA, Bloomfield P. The medical management of valvar heart disease. Heart 2002; 87:395-400. 